Mon. Not. R. Astron. Soc. 000,19() Printed 18 January $2021 \quad$ (MN LATEX style file v2.2)

\title{
Scintillation observations of PSR B0823+26
}

\author{
M. Daszuta` W.Lewandowski J. Kijak \\ Kepler Institute of Astronomy, University of Zielona Gora, Lubuska 2, PL-65-265 Zielona Gora, Poland
}

Accepted ...; Received ...; in original form

\begin{abstract}
We present results of the analysis of interstellar scintillation in PSR B0823+26. Observations were conducted at a frequency of $1.7 \mathrm{GHz}$ using the 32-m Torun Centre for Astronomy radio telescope. More than 50 observing sessions, lasting on average $10 \mathrm{~h}$, were conducted between 2003 and 2006. We found interstellar scintillation parameters by means of dynamic spectrum analysis as well as structure function analysis of the flux density variations. We identified two distinctive time-scales, which we believe to be the time-scales of diffractive and refractive scintillation. Our results show that at the given frequency the diffractive time-scale in PSR B0823+26 is $\tau_{\text {diss }}=19.3_{-1.6}^{+1.7}$ min, the refractive time-scale is $\tau_{\text {riss }}=144 \pm 23 \mathrm{~min}$ and the decorrelation bandwidth is $B_{\text {iss }}=81 \pm 3 \mathrm{MHz}$.
\end{abstract}

Key words: pulsars: general - pulsars: individual: PSR B0823+26 -ISM:structure.

\section{INTRODUCTION}

The theory of the propagation of radio waves through the interstellar medium is constantly evolving. The plasma modulation phenomena affecting radio waves can be described by means of the three-dimensional spatial power spectrum ' of electron density fluctuations, which is described by a Kolmogorov spectrum (Rickett 1990):

$P_{3 n}=C_{n}^{2} q^{-\beta}$,

where $C_{n}^{2}$ is a measurement of the mean turbulence of the electron density along the line of sight and $q=2 \pi / s$ is the wavenumber associated with the spatial scale of turbulence $s$. This formula holds, assuming that the spatial scale $s$ is between the inner and outer scales of the spatial density fluctuations $\left(s_{\text {inn }} \ll s \ll s_{\text {out }}\right)$. The spectral index $\beta$ is believed to be in the range 3-5 (see also Romani, Narayan \& Blandford 1986). Kolmogorov theory, which describes the propagation of turbulent energy from large scales to small scales, gives the expected value of $\beta=11 / 3$.

The theory of interstellar scintillation (ISS) was developed by Scheuer (1968). The simplest model commonly used for the explanation of scintillation and scattering effects is the thin screen model. Although it was shown for the case of several lines of sight that this model does not explain the observed scintillation behaviour (see for example Bhat. Gupta \& Rao 1999), its simplicity allows for easy understanding of these phenomena as well as an interpretation of the observational results. According to this model, the irregularities of the interstellar medium are located within

\footnotetext{
* E-mail: muzzy@astro.ia.uz.zgora.pl (MD)
}

a thin screen, which is located midway between the pulsar and the observer. Scattering within the screen causes the signal to be spread over a time interval $\tau_{s}$ (called the pulse broadening time) and hence shows a variety of phases over a range $\delta \phi \sim 2 \pi f \tau_{s}$. Phase modulation of the signal produces interference patterns at the observers plane.

Depending on the size of the wavefront perturbations, one can distinguish two types of interstellar scintillations, the so-called weak and strong scintillation regimes. The strong scintillations can be divided into two different branches: diffractive interstellar scintillations (DISS), arising from small-scale fluctuations $\left(10^{6}-10^{8} \mathrm{~m}\right.$ : Cordes, Weisberg \& Boriakoff 1985) and refractive scintillations (RISS), which form due to large-scale irregularities $\left(10^{10}-10^{13} \mathrm{~m}\right.$ : Sieber 1982$)$.

The scintillations can be characterized by three basic parameters: the scintillation time-scale $\tau_{\text {iss }}$, the decorrelation bandwidth $B_{\text {iss }}$ and the modulation index $m$, which is the ratio of root-mean-square (RMS) deviation of the observed flux densities $F$ to the mean value of the time series (Lorimer \& Kramer 2005):

$m=\frac{\sqrt{\left\langle(F-\langle F\rangle)^{2}\right\rangle}}{\langle F\rangle}$.

The decorrelation bandwidth is related to the scatter by the relation: $2 \pi \tau_{\text {iss }} B_{\text {iss }}=C_{1}$, where $C_{1}$ is often assumed to be close to unity, although it may vary for different geometries and/or models (Lambert \& Rickett 1999).

Another useful parameter, the scintillation velocity $V_{\text {iss }}$ is a value describing the velocity at which the observer passes through the scintillation interference patterns. The scintillation velocity combines the transverse pulsar velocity, the 
Table 1. PSR B0823+26 scintillation parameters at multiple frequencies. We present two values of $V_{i s s}$ : the one originally published and the recalculated one (marked by an asterisk) obtained using the proper values of the pulsar distance and the scintillation velocity constant $A_{v}$. Values without uncertainties quoted were presented in such a way by the original authors.

\begin{tabular}{|c|c|c|c|c|c|c|c|c|c|c|}
\hline $\begin{array}{l}\text { Freq. } \\
(\mathrm{MHz})\end{array}$ & $\begin{array}{l}B_{\text {diss }} \\
(\mathrm{MHz})\end{array}$ & $\begin{array}{l}\tau_{\text {diss }} \\
(\min )\end{array}$ & $\begin{array}{l}\tau_{\text {riss }} \\
(\min )\end{array}$ & $\begin{array}{c}V_{i s s} \\
\left(\mathrm{~km} \mathrm{~s}^{-1}\right)\end{array}$ & $\begin{array}{c}V_{i s s}^{*} \\
\left(\mathrm{~km} \mathrm{~s}^{-1}\right)\end{array}$ & $\mathrm{u}$ & $\begin{array}{c}\theta_{d} \\
(\mathrm{mas})\end{array}$ & $\begin{array}{c}\theta_{r} \\
(\mathrm{mas})\end{array}$ & $\begin{array}{c}\log C_{n}^{2} \\
\left(\mathrm{~m}^{-20 / 3}\right)\end{array}$ & $\alpha$ \\
\hline $74^{a}$ & - & - & $12^{\mathrm{d}} \pm 6$ & 365 & - & - & - & - & - & - \\
\hline $327^{b}$ & $0.293 \pm 0.041$ & $2.1 \pm 0.31$ & 5254 & - & $277 \pm 16$ & $47.8 \pm 0.8$ & $1.01 \pm 0.02$ & $0.01 \pm 0.03$ & $-3.24 \pm 0.03$ & - \\
\hline $408^{c}$ & 0.26 & 3.3 & 3456 & 140 & 149 & 34 & - & - & - & - \\
\hline $430^{d}$ & $0.39 \pm 0.07$ & - & - & - & - & - & - & - & -3.64 & 4.4 \\
\hline $1000^{e}$ & 33 & 11 & - & $241 \pm 72$ & $206 \pm 61$ & - & - & - & - & - \\
\hline $1540^{f}$ & $82 \pm 5$ & $13.6 \pm 8$ & 576 & - & $187 \pm 9$ & 6 & $0.070 \pm 0.002$ & $0.004 \pm 0.005$ & -2.8 & 3.9 \\
\hline $1700^{g}$ & $81 \pm 3$ & $19.3_{-1.6}^{+1.7}$ & $144 \pm 23$ & - & $108 \pm 21$ & 2.7 & $0.065 \pm 0.002$ & 0.005 & -2.67 & $3.94 \pm 0.36$ \\
\hline $4.75^{h}$ & - & $9 \pm 1$ & - & - & - & - & - & - & - & - \\
\hline $10.55^{h}$ & - & $7.5 \pm 2$ & - & - & - & - & - & - & - & - \\
\hline
\end{tabular}

${ }^{a}$ Gupta, Rickett \& Coles (1993),${ }^{b}$ Bhat, Gupta \& Rao (1999), ${ }^{c}$ Smith \& Wright $(1985),{ }^{d}$ Cordes, Weisberg \& Boriakoff (1985), ${ }^{e}$ Gupta (1995),

${ }^{f}$ Wang et al. (2005), ${ }^{g}$ our results, ${ }^{h}$ Malofeev et al. (1996)

velocity of the Earth's transverse motion and the intrinsic screen velocity. This value can be estimated using other scintillation parameters as:

$V_{i s s}=A_{\mathrm{V}} \frac{\sqrt{d_{\mathrm{kpc}} B_{\mathrm{iss}, \mathrm{MHz}} X}}{f_{\mathrm{GHz}} \tau_{\mathrm{iss}, \mathrm{sec}}}$,

where constant $A_{\mathrm{V}}$ depends on geometry. In our calculations, following Gupta. Rickett \& Lyne (1994), we used the value $A_{V}=3.85 \times 10^{4} \mathrm{~km} \mathrm{~s}^{-1}$. Here, $d_{\mathrm{kpc}}$ is the pulsar distance and $X$ is the ratio of the screen-observer distance to the screen-pulsar distance. For a thin screen located midway between pulsar and observer, one obtains $\mathrm{X}=1$.

In dynamic spectrum analysis, one can often observe the sloped patterns (Hewish, Wolszczan \& Graham 1985; Bhat, Gupta \& Rao 1999) that arise from refraction. These sloped fringes can be characterized by the frequency drift rate $d t / d \nu$, which can be related to the refractive scattering angle $\theta_{r}$ by the following expression:

$\frac{d t}{d \nu}=\frac{\theta_{r} d_{\mathrm{kpc}}}{V_{\mathrm{iss}} f_{\mathrm{GHz}}}$.

In this article, we present the results of our analysis of ISS observations of PSR B0823+26, at a frequency of 1.7 $\mathrm{GHz}$. Our observations were longer by a factor of four than any other observing project conducted for this pulsar in the past, since we had over $600 \mathrm{~h}$ of integration time for this pulsar.

\section{OBSERVATIONS AND DATA ANALYSIS}

The observations were conducted at a frequency of $1.7 \mathrm{GHz}$ using the 32-m Toruń Centre for Astronomy radio telescope. The total intensity signal was obtained using the Penn State Pulsar Machine II (PSPM II:(Konacki et al. 1999)), which is basically a 64-channel filter-bank spectrometer with a total bandwidth of $192 \mathrm{MHz}$. Calibration of the signal was performed by injecting the noise-diode signal to our receiver, which was carried out synchronously with the pulsar period. At the start and end of each observing session, the noise diode signal itself was calibrated by means of total intensity observations of a well-known radio source 3C393.1.

The PSPM II output (in the observing mode we used) was basically 64 time-integrated pulse profiles corresponding to the backend spectral channels. This allowed us to perform both dynamic spectrum analysis and after the dedispersion process and an offline integration of the whole bandwidth total intensity (pulsar average flux density) measurements.

Observations were conducted between 2003 and 2006. We gathered data during 70 observing sessions, consisting of 5 min individual integrations. More than 50 of those sessions lasted for $10 \mathrm{~h}$ or more; there were also shorter sessions (lasting less than $6 \mathrm{~h}$ ) and the longest sessions lasted for more than $12 \mathrm{~h}$. The total integration time of all our observations analysed for the purpose of this article was $\sim 600 \mathrm{~h}$.

The pulsar we observed was PSR B0823+26. This is relatively well-studied source, but its scintillation properties have never before investigated in such a long-term extensive observing project. It is a typical, $530-\mathrm{ms}$ period pulsar, which is relatively close at a distance of 0.38 kpc (Gwinn et al. 1986), with a dispersion measure of $D M=19.4 \mathrm{pc} \mathrm{cm}^{-3}$. The scintillation parameters of the PSR B0823+26 have been measured previously by many authors at various frequencies, ranging from $74 \mathrm{MHz}$ to 10.55 $\mathrm{GHz}$ (see Table 1 for a full list of references).

\section{$2.1 \quad$ Flux density measurements}

The bottom panel of Fig. 1 presents the flux density averaged over the individual observing sessions versus observing epoch. As one can see, single-session average values range from $\sim 1 \mathrm{mJy}$ to over $30 \mathrm{mJy}$. To estimate the errors of the average flux measurements we used two methods. First we used an equation that considers the physical scintillation effects (Kaspi \& Stinebring 1992):

$\frac{\delta F}{F} \simeq \frac{m_{\text {riss }}}{\sqrt{T_{\text {obs }} / \tau_{\text {riss }}}}$,

where $T_{o b s}$ is the total observation time, $\tau_{\text {riss }}$ is refractive timescale (we used $\tau_{\text {riss }}=144 \mathrm{~min}$, see the following sections) and $m_{\text {riss }}=0.6$ is the expected RISS modulation index (based upon Lorimer \& Kramer 2005: $m_{\text {riss }}=$ $\left.\left(B_{i s s} / f\right)^{1 / 6}\right)$. For the second method, we estimated RMS for each individual session (with the receiver/calibration noise factor included), which turned out to be up to 30 per cent of the average flux value. As a final uncertainty estimate for a given observing session we used the greater of the two values; these are shown as error bars in the bottom panel of Fig. 1 . We also calculated the total average flux value based on all 


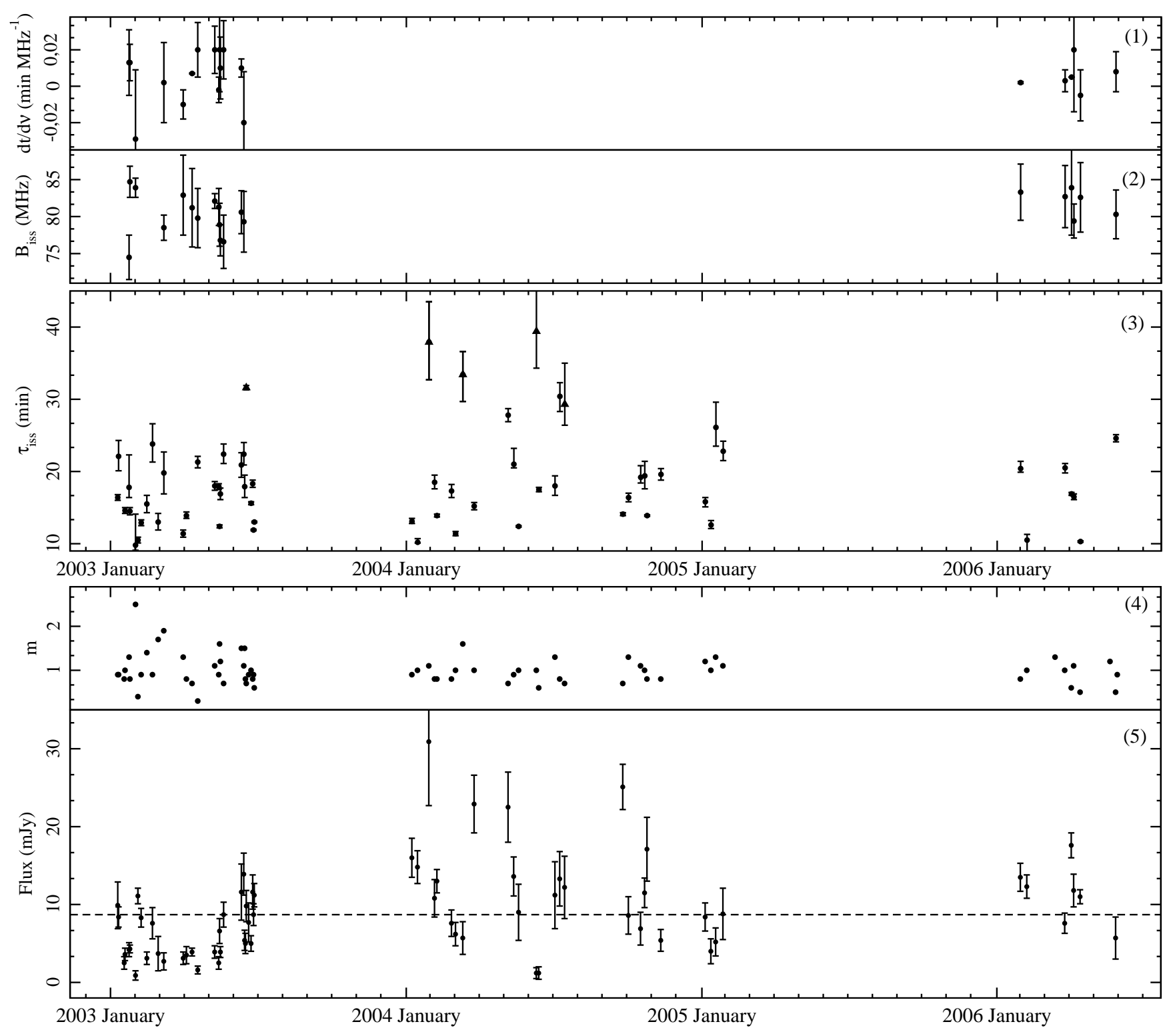

Figure 1. Plots of the time series of scintillation parameters and the PSR B0823+26 flux density. The drift rate $(d t / d \nu$, panel $(1))$ and the decorrelation bandwidth $\left(B_{i s s}\right.$, panel $\left.(2)\right)$ are derived from the dynamic spectrum analysis, while the scintillation time-scale $\left(\tau_{d i s s}\right.$, panel (3)) is derived from the structure function analysis. Triangles in the diffractive time-scale plot denote those measurements we do not consider reliable, due to confusion with the refractive time-scale. The last two plots show the modulation index $(m$, panel $(4))$ and session-average flux density $(F$, panel(5)). The uncertainties in the measurements indicate $\pm 2 \sigma$ and in the case of the flux density they were either calculated using equation (4) or using the time series RMS (whichever value was greater).

available individual integrations, which yielded a value of $\langle F\rangle=8.7 \pm 1.5 \mathrm{mJy}$. The flux density is strongly modulated in the individual sessions: panel (4) in Fig. 11 shows the value of the modulation index (obtained using the formula given in equation 10.

The variation of the flux density during individual sessions usually showed the expected quasi-periodic fluctuations caused by the scintillations; however, in a few of our observing sessions we found a characteristic disappearance of the pulsar signal. The upper panel of Fig. 2 shows an example of a light curve with a flat-bottomed minimum between 150 and $250 \mathrm{~min}$. A similar suppression in quasar light curves was observed by Clegg. Fev \& Lazio (1998). They be- lieve that the extinction of the flux corresponds to inhomogeneities of the interstellar medium in the form of a plasma lens (with a Gaussian distribution of density of free electrons) crossing the line of sight of the quasar (or, in principle, any point source). The disappearance of the source would be accompanied by a caustic increase of the flux just prior to and immediately after the minima, due to the increase in flux caused by the reflection of radiation by the incoming/outgoing plasma lens. A detailed study of such occurrence could allow for estimation of the size and possibly velocity of such plasma lenses; however, we found our data to be insufficient for that purpose. Only in one case were we able to observe the full extent of such an event, 

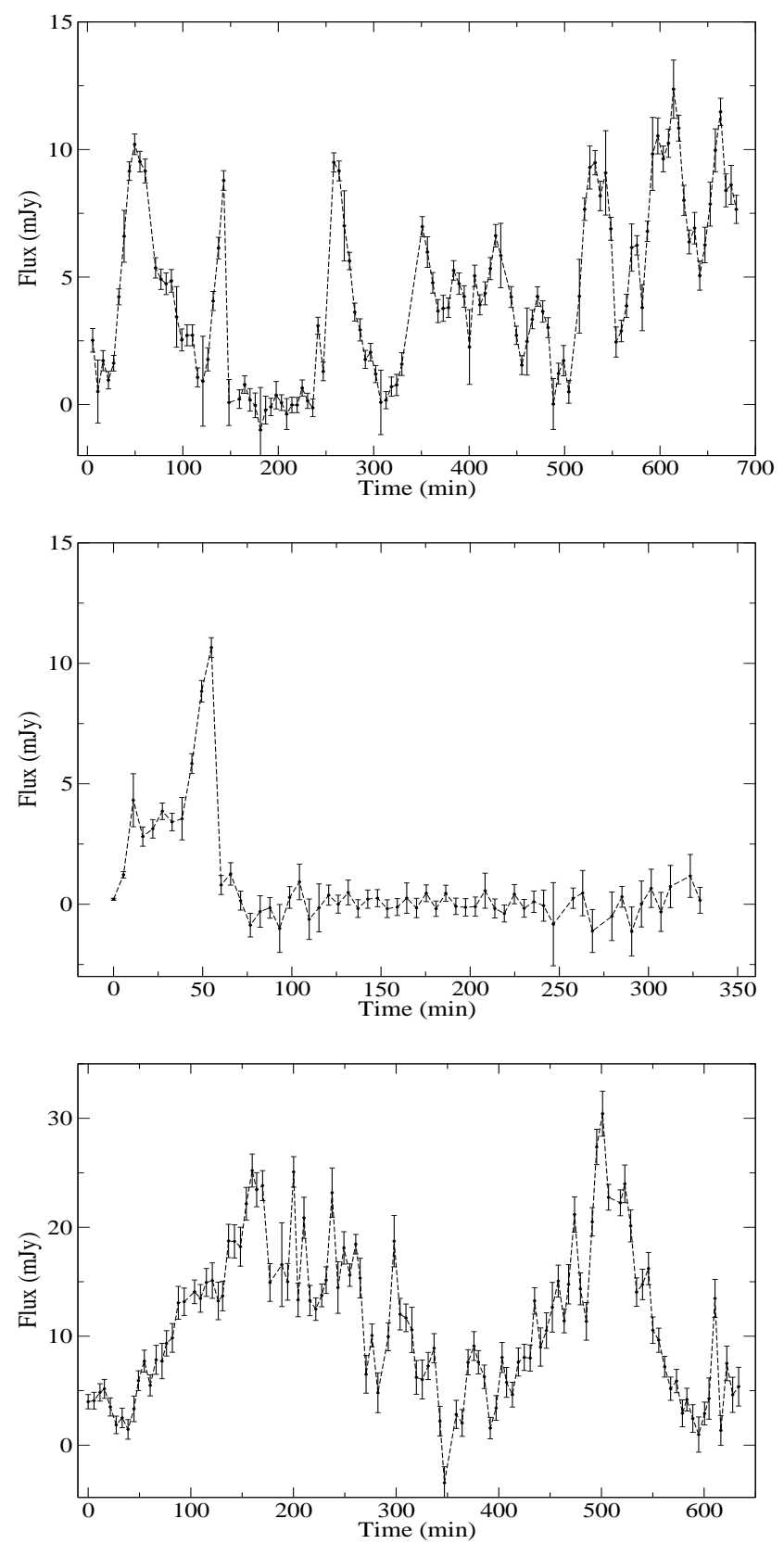

Figure 2. Flux density variations of PSR B0823+26 during selected individual observing sessions. The first plot shows a light curve with characteristic flat-bottomed minimum with a duration of $100 \mathrm{~min}$ (2003 January 23). The middle plot shows another session where the pulsar flux density increases and then suddenly vanishes completely for the reminder of the session (2003 January 31 ). The bottom plot shows an example of a session with a long ( $200 \mathrm{~min}$ ) time-scale clearly visible (2003 June 27). Errorbars for the single-integration flux measurements include instrumental effects.

lasting for $\sim 2 \mathrm{~h}$ (top panel of Fig. 2), but this particular observation is strongly affected by diffractive scintillations, which make finding the flux baseline - and hence the whole Clegg. Fev \& Lazio (1998) model - almost impossible. We also noted a few cases where the pulsar disappeared during the session (with a flux increase just prior to it) and was not detectable for the reminder of the session, i.e. the disappearance was at least 6-7 h long (see the bottom panel in Fig. 2). We also detected one case where the pulsar was not visible at the beginning of the session but then suddenly appeared after a few hours (apparently during a single 5 minute integration) with a significant flux density value.

Overall, such occurrences appear not to be very common for PSR B0823+26 (only four of our 70 sessions); when such an occurrence appears, its duration is from $2 \mathrm{~h}$ up to intervals comparable to the length of our observing session, or possibly longer.

\subsection{Analysis of dynamic spectra}

The dynamic spectrum is a measure of the intensity of the pulsar at multiple frequencies over time. This is best described via a two-dimensional image of the pulse intensity as a function of time and frequency. The pulsar is a source emitting coherent radiation; diffraction and refraction in the interstellar medium disturb the wavefront, which leads to self-interference, amplifying or suppressing the pulsar signal as a result. This can be seen in the dynamic spectrum as changing bright and dark bands or patches, which form a pattern of diffractive scintillations of a pulsar (see for example Cordes. Pidwerbetsky \& Lovelace 1986). A single pattern (enhanced region of flux density) in a dynamic spectrum is called a scintle.

Using the dynamic spectrum, one can estimate the decorrelation bandwidth $B_{\text {iss }}$, decorrelation (diffractive) time-scale $\tau_{\text {iss }}$ and drift slopes $d t / d \nu$. The values of the scintillation parameters are determined by fitting a twodimensional Gaussian function to the dynamic spectrum auto-correlation function. Using standard given by Cordes (1986), we define the decorrelation bandwidth $B_{\text {iss }}$ as the half-width at half maximum of the intensity auto-correlation function. The scintillation time-scale $\tau_{i s s}$ is defined as the half-width at $1 / e$ along the time lag axis. The drift rate is a measure of the slope of the line joining the points on the ellipse with the highest correlation at a given frequency offset (Gupta. Rickett \& Lvne 1994) and can be determined by finding the inclination angle of the fitted twodimensional Gaussian. We observed 20 dynamic spectra of PSR B0823+26. Fig. 4] shows a sample of our dynamic spectra. The $x$-axis represents the frequency (64 spectral channels, each $3 \mathrm{MHz}$ wide, centered at $1.7 \mathrm{GHz}$ ), and the $y$-axis is the epoch quantified by the 5-minute length of a single integration.

At the time of our observations the telescope was affected by a wide range of radio interference, both narrowband persistent features, which basically destroyed all the data in several spectral channels of PSPM II, and shorttime wide-bandwidth interference, capable of disturbing an individual integration over the entire observing bandwidth. Most interference cleaning was performed in the offline analysis process.

Since the expected variations in the dynamic spectrum due to the scintillation phenomenon should be rather smooth, then any sharp or abrupt change in the measured flux density (with regard to both time and observing frequency) can be attributed to radio interference. We removed such measurements from our dynamic spectra and replaced 
them by values interpolated from the adjacent measurements, which were not affected by radio interference.

The results of our analysis of dynamic spectra are shown in Fig. 11 (top two panels). We also calculated the average values of the diffractive time-scale, $\tau_{i s s}=19 \pm 3 \mathrm{~min}$, the decorrelation bandwidth, $B_{i s s}=81 \pm 3 \mathrm{MHz}$, and the drift rate, $d t / d \nu=0.027 \pm 0.013 \min \mathrm{MHz}^{-1}$.

\subsection{Structure function analysis}

Structure function analysis is used to study interstellar scintillation and turbulence in random media, as it is an useful tool to estimate the characteristic timescales of the variability present in the data. We obtained structure function following the method described by Simonetti. Cordes \& Heeschen (1985). The structure function displays three characteristic regimes.

(i) The noise regime at small lags, dominated by noise and correlated variation of the time series. The time lags are much shorter than the shortest time-scale present in random process.

(ii) The structure regime, characterized by a monotonic increase, showing a slope in a log-log plot.

(iii) The saturation regime, where structure function flattens.

The characteristic time-scale of the variations can be found as the time lag at which the value of the structure function is equal to half the saturation value, $D\left(\tau_{c}\right)=D_{\text {sat }} / 2$. Applied to the flux density time series, the structure function analysis can be used to estimate the scintillation time-scale, as well as the modulation index, by means of the formula given by (Kaspi \& Stinebring 1992):

$m=\sqrt{D_{\text {sat }} / 2}$.

The error estimates for the time-scale and the modulation index can be found, assuming that the saturation level uncertainty is $\delta D_{\text {sat }} / D_{\text {sat }} \approx\left(2 \tau_{c} / T_{\text {obs }}\right)^{1 / 2}$, where $T_{\text {obs }}$ is the total duration of the observing session.

We analyzed more than 70 observing sessions using the structure function analysis. Fig. 4 shows typical structure function plots, obtained from individual sessions. Horizontal dashed lines on the plot show the saturation level (with the appropriate error estimates marked), while the vertical lines correspond to the characteristic time-scales found (again, the error bar crossing the dashed line represents the timescale error estimate). For some of our observing sessions, we were unable to find the scintillation time-scale due to a lack of saturation in the structure function. The results of our measurements of the diffractive time-scale obtained from individual sessions are shown in Fig. 1 (panel 3).

For some of the sessions we were able to detect two plateaus in the structure function plots (see the bottom plot in Fig. (4), which can be attributed to two separate timescales present in the data. In such cases, the values of $\tau_{\text {diss }}$ obtained for the shorter time-scale are shown on the subplot (3) and we believe these correspond to the DISS, which (based on the previously published results) at our observing frequency of $1.7 \mathrm{GHz}$ should be of the order of $\sim 20 \mathrm{~min}$. The average diffractive time-scale calculated from all the data gathered is $\tau_{\text {diss }}=19.3_{-1.6}^{+1.7} \mathrm{~min}$. The values marked by
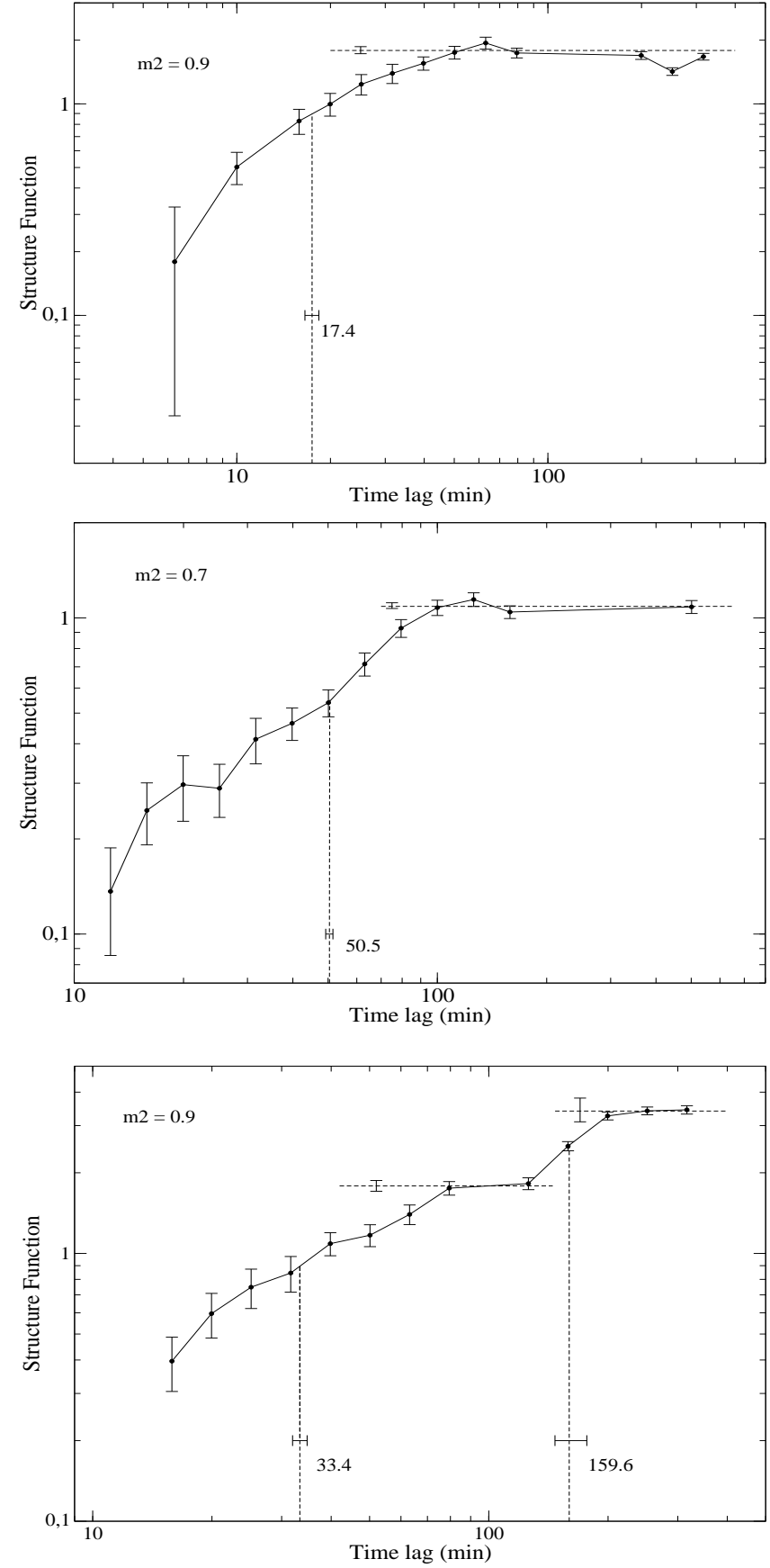

Figure 4. Structure function plot of the flux density time series for two of the individual sessions from our project: 2004 February 26 (top), 2003 June 27 (middle) and 2003 January 18 (bottom). The horizontal dashed line on the plot shows the position of the saturation plateau and the vertical line indicates the value of the scintillation time-scales; $\mathrm{m} 2$ denotes the value of the flux density modulation index. Uncertainties in the structure function values were calculated as $\sigma_{D}(i)=\sigma_{\mathrm{WN}} \sqrt{8 D(i) / N(i)}$ (where $\sigma_{\mathrm{WN}}$ is the white noise: Simonetti. Cordes \& Heeschen 1985)

triangles on the same subplot are time-scale derived from the second plateau detected in the structure function.

We believe that the second plateau appeared in some of our structure function plots due to the refractive scintillation time-scale. However, since its value is relatively close to the total length of the observing session, the value may be 

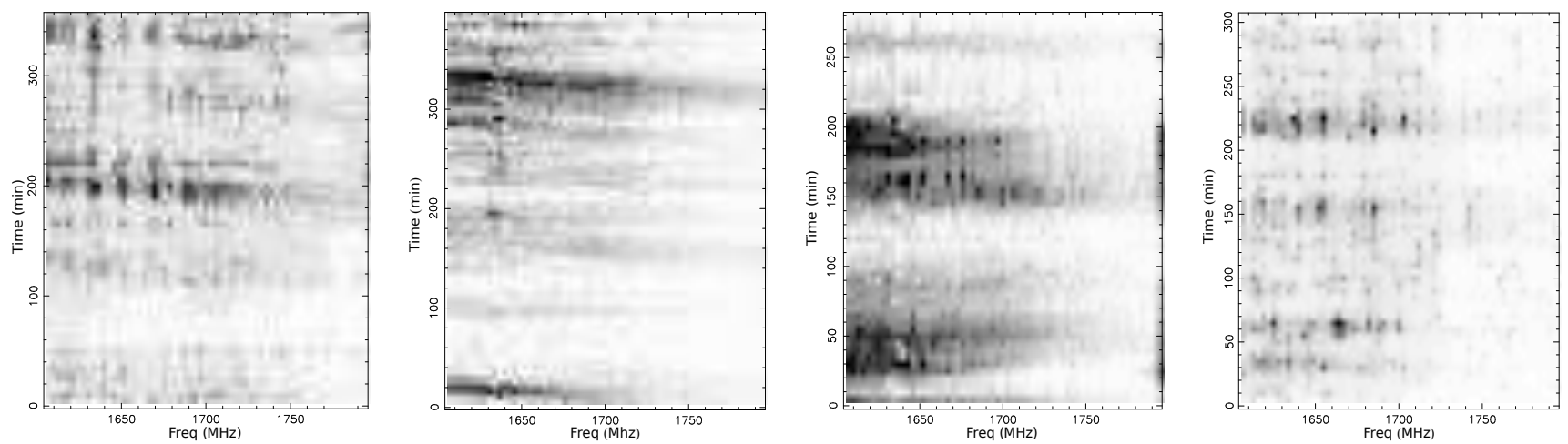

Figure 3. Four example dynamic spectra of PSR B0823+26. The intensity variations of pulsar radiation are presented in the form of gray-scale plots (where a darker shade of gray indicates greater intensity). Starting from the left panel, plots are from 2003 April 18 , 2003 May 9, 2003 May 20 and2006 April 14.

distorted due to insufficient sampling of the structure function at very long time lags. Nevertheless, we were able to obtain the value of the time-scale corresponding to the second plateau for five observing sessions, ranging from 108-196 min, with an average of $144 \mathrm{~min}$, which we believe to be a structure function estimate of the refractive time-scale.

One has to note, however, that in some cases the structure function method did not manage to find the second time-scale, despite the fact that it is clearly visible by the naked eye in the flux time series. This is best illustrated by the structure function presented in the bottom panel of Fig. 4. which is the result of applying the analysis to the time series from the 2003 June 27, shown in the middle panel of Fig. 2. One can clearly see that the flux is strongly modulated by - supposedly - refractive scintillations, with a timescale of $\sim 200 \mathrm{~min}$, yet the structure function shows only a single, very broad plateau. Even if we were able to see the refractive time-scale plateau - which should be somewhere behind the right edge of the plot (but was heavily distorted due to insufficient sampling) - its time-scale, clearly visible by eye, would fall within the plateau shown, making the time-scale measurement futile. Hence, for a comparison, we decided to use a simpler method of flux time series autocorrelation function analysis to estimate the value of the refractive scintillation time-scale. Our results, based on a the few best time series where the refractive scintillations were visible, yielded the average value of $\tau_{\text {riss }}=170 \pm 13 \mathrm{~min}$. This may suggest that the value of the refractive time-scale from structure function analysis (cited in the previous paragraph) may be underestimated. It seems that the refractive timescale is varying a lot, and since structure function method is unable to detect longer time-scales, the average value obtained from it may be prone to selection effects.

\section{RESULTS AND DISCUSSION}

The theory of the interstellar scintillation predicts that with increasing observing frequency the diffractive timescale increases, while the refractive time-scale decreases. When observing at the so-called critical frequency, both time-scales become equal and the scintillations behaviour changes from the strong to weak regime (Rickett 1990). This is best described by the strength of scattering parameter $u$
( $u>1$ for strong scintillations), which can be estimated as Lorimer \& Kramer (2005)

$u^{2}=\tau_{\text {riss }} / \tau_{\text {diss }}$.

Using the value of $\tau_{\text {diss }}$ from the structure function analysis, as found from a larger number of observing sessions, we calculated $u=2.7$.

The strength of the scattering parameter is defined in scintillation theory (Rickett 1990) as $u=r_{F} / s_{0}$, where $r_{F}$ is the Fresnel scale and $s_{0}$ is the coherence scale. The latter, in principle, cannot be obtained from observation; one can, however, estimate diffractive and refractive scales. Estimation of the diffractive scale can be made using the value of the scintillation velocity. Using equation 2 and our results described in the previous section we obtained that $V_{\text {iss }}=108 \pm 21 \mathrm{~km} \mathrm{~s}^{-1}$. The scintillation velocity can be used to estimate the diffractive scale $\left(s_{d}=V_{\text {iss }} \tau_{\text {diss }}\right)$, which yielded $s_{d}=1.25 \times 10^{8} \mathrm{~m}$.

Following Bhat. Gupta \& Rao (1999), the diffractive angle can be expressed as $\theta_{d}=\left(c / \pi d B_{\text {iss }}\right)^{1 / 2}$, which, using our results, resulted in $\theta_{d}=0.065$ mas. Using this value, we can estimate the refractive scale $\left(s_{r} \simeq d \theta_{d}\right)$ as $s_{r}=3.71 \times 10^{9}$ $\mathrm{m}$. This allows us to estimate the Fresnel scale $\left(r_{F}^{2}=s_{d} s_{r}\right)$, which in the case of B0823+26 is $r_{F}=6.8 \times 10^{8} \mathrm{~m}$.

\subsection{Spectrum of plasma density fluctuations}

Gathering the data from various observing frequencies allows us to study the frequency dependence of the scintillation parameters. The theory of interstellar turbulence predicts that the scintillation parameters depend strictly on the observing frequency (Romani, Naravan \& Blandford 1986). Table 2 shows predicted values based various models, as well as our results. Using previously published values (Table 1) and adding our measurements, we estimated the spectral slope for the scintillation time-scale and decorrelation bandwidth (see Fig. 5).

As discussed before, we used value of the DISS timescale obtained from structure function analysis. A linear fit to the diffractive time-scale versus the observing frequency data yielded $\tau_{\text {diss }} \sim f^{1.25 \pm 0.1}$. This is very close to the value expected for Kolmogorov-type turbulence $(\sim 1.2)$. A similar fit for the decorrelation bandwidth versus the frequency dependence yielded $B_{\text {diss }} \sim f^{3.94 \pm 0.36}$. One has to note, 


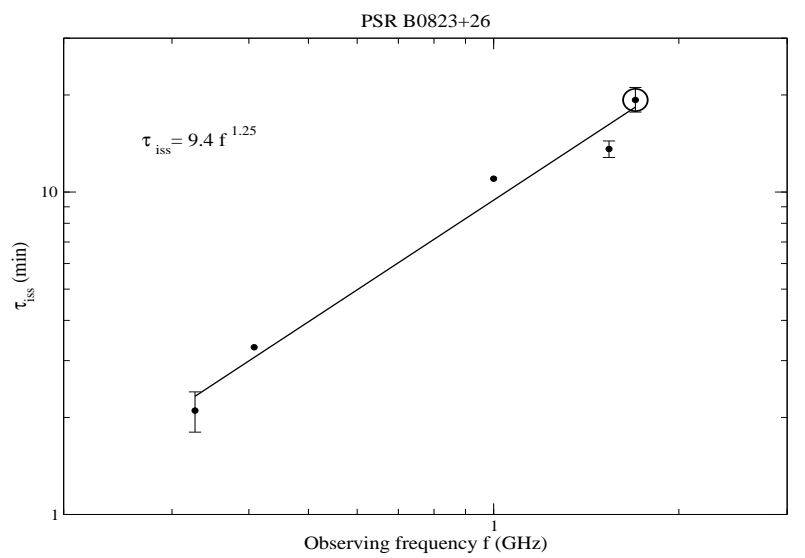

Scintillation observations of PSR B0823+26

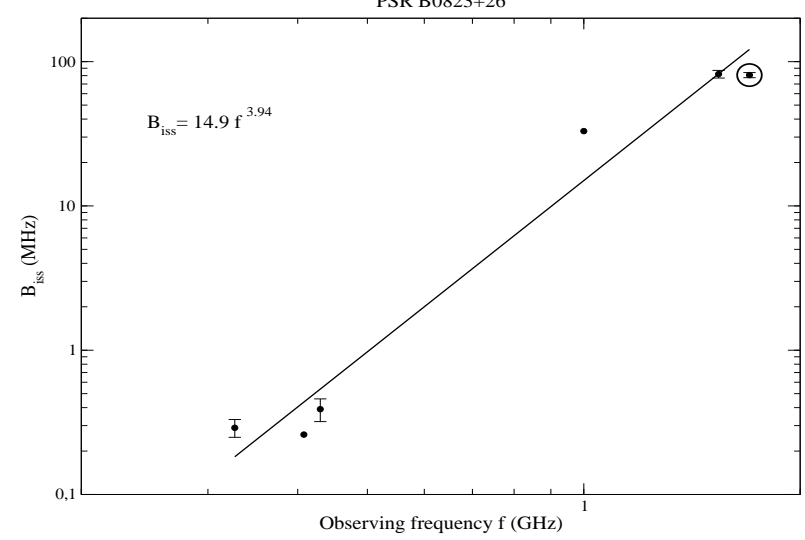

Figure 5. Left: a plot of diffractive time-scale versus observing frequency based on data found in the literature and our measurements at $1.7 \mathrm{GHz}$ (circle in the ring). Right: a similar plot for the decorrelation bandwidth. For the references for the lower frequency values, see Table 1 Solid lines represent our fits of the spectral indices (results for which are shown in the upper left part of each plot). Note that some of the values taken from the literature did not have their uncertainties given.

however, that in both cases a proper uncertainty analysis could not be performed, as for some of the previously published values we could not find the error estimates. Therefore we believe that the decorrelation bandwidth spectral index, although formally inconsistent with the simple model predictions (the value of 4.4 is outside of error estimate), may be marginally considered to be compliant with the Kolmogorov's theory thin-screen model.

The last column of Table 1 also includes three values of the decorrelation bandwidth spectral indices published earlier. Smith \& Wright (1985) obtained their value from an independent method: scatter-broadening measurements. Cordes, Weisberg \& Boriakoff (1985) obtained the value from a fit for decorrelation bandwidth (similar to ours, but with fewer data available at the time, only two measurements), and their result is consistent with a Kolmogorov spectrum $(\alpha=4.4)$. The estimation of Wang et al. (2005), as well as our estimate is rather closer to the critical spectrum $(\beta=4)$ than Kolmogorov one.

Deviations from Kolmogorovs model predictions are not uncommon: see (Lewandowski et al. 2013) for a recent summary based on the pulse-broadening observations. In the case of the scintillation studies, Bhat, Gupta \& Rao (1999) showed cases of non-compliance with the simple thinscreen Kolmogorov model in their analysis of 18 objects and more recently Lewandowski et al. (2011) provided similar results for PSR B0329+54, a pulsar that is quite similar to B0823+26 when it comes to the distance and dispersion measure.

Based on a model for the local interstellar medium, we know that the PSR B0823+26 is outside the shell of the Local Bubble (Bhat, Gupta \& Rao 1998). Following Cordes \& Lazio (2002), the pulsar is also outside the Loop $I$, which is also apparently reflected in its interstellar scattering properties (Bhat \& Gupta. 2002). This could suggest that the interstellar medium density pattern along the PSR B0823+26 line of sight is indeed completely different from the single thin-screen model, which would explain the possible non-compliance with its predictions.

Using both scintillation time-scales and their frequency dependence (inferred from the fits to the whole observed frequency range data), we were able to estimate that for
Table 2. The predicted and observed spectral index of frequency dependencies for the diffractive time-scale and the decorrelation bandwidth.

\begin{tabular}{cccc}
\hline \multicolumn{3}{c}{ Predicted spectral index by theory } & Our results \\
,steep" & ,critical” & Kolmogorov & \\
4.3 & 4 & $11 / 3$ & \\
\hline$B_{\text {iss }}+4.7$ & +4 & +4.4 & $3.94 \pm 0.36$ \\
$\tau_{\text {diss }}+1.4$ & +1 & +1.2 & $1.25 \pm 0.1$ \\
\hline
\end{tabular}

PSR B0832+26 the critical frequency is $f_{c} \sim 5 \mathrm{GHz}$ and at this frequency one expects a transition from strong to weak scintillation. Malofeev et al. (1996) studied the scintillation parameters at $4.75 \mathrm{GHz}$ and $10.55 \mathrm{GHz}$. Using the structure function analysis - they measured $\tau_{\text {iss }}=9$ and $\tau_{\text {iss }}$ $=7.5 \mathrm{~min}$, respectively. They also suggested that at these observational frequencies the pulsar switched to the weak scintillation regime.

We have also calculated the level of turbulence (using the formula from Cordes 1986) to be $C_{n}^{2}=-2.67$; this value determines the average electron density fluctuation that produce scattering.

\subsection{Scintillation velocity}

The proper motion and the heliocentric parallax measurements of PSR B0823+26 provide us with a good estimate of both the pulsar distance and its transverse velocity, which is $V_{p m}=194 \pm 41 \mathrm{~km} / \mathrm{s}$ (Lyne, Anderson \& Salter 1982).

Table 1 also shows the results for the scintillation velocity, which should be close to the pulsar transverse velocity. In the past, various authors used a different distance and the $A_{v}$ constant (see references) to determine $V_{i s s}$. We generalized their measurements (Table 1] sixth column) and recalculated the scintillation velocity using the proper values of the distance $d=0.38 \mathrm{kpc}$ and the constant $A_{v}=3.85 \times 10^{4}$ (see eq. 2). For our data we estimated $V_{i s s}=108 \pm 21 \mathrm{~km} / \mathrm{s}$. In principle, the values of velocity should be similar, regardless of the observing frequency. However, even after the recalculation one can still note major differences.

One possible explanation may be that our value comes 
for the grand average of both our DISS time-scale measurements and our decorrelation bandwidth measurements, an average from $\sim 600 \mathrm{~h}$ of integration. However, if we take a values of both $\tau_{\text {diss }}$ and $B_{\text {iss }}$ from some of our individual sessions, we can get a result similar to the proper motion velocity and close to the value of $220 \mathrm{~km} / \mathrm{s}$, which would be in better agreement with the previously published results. Such a discrepancy, especially when it comes from a single epoch measurements, may arise from the fact that the scintillation velocity is a vector sum of pulsar proper motion, the Earth's orbital motion $V_{E}$ and the bulk flow of the intensity irregularities $V_{i r r}$. Depending on the epoch and the screen location, $V_{E} \sim$ may add up to $30 \mathrm{~km} / \mathrm{s}$ while $V_{i r r}$ is usually less than $10 \mathrm{~km} / \mathrm{s}$ (Bhat, Gupta \& Rao 1999).

\section{SUMMARY AND CONCLUSIONS}

In this article we have shown the results of long-term observations of the pulsar PSR B0823+26 at a frequency of 1.7 GHz. The total integration time of all observations we used was $\sim 600 \mathrm{~h}$, which makes our project the most extensive study of this pulsar's scintillation parameters. Using analysis of dynamic spectra, we found average interstellar scintillation parameters, such as time-scale $\tau_{\text {diss }}=19 \pm 3$ min and decorrelation bandwidth $B_{\text {diss }}=81 \pm 3 \mathrm{MHz}$. We also observed characteristic drift patterns with a drift rate of $d t / d \nu=0.027 \pm 0.013 \mathrm{~min} \mathrm{MHz}^{-1}$, which indicates the presence of refractive scintillations. Using the values of $\tau_{\text {diss }}$ and $B_{d i s s}$, we estimated the scintillation velocity to be $V_{i s s}$ $=108 \pm 21 \mathrm{~km} / \mathrm{s}$. Our analysis using the structure function method, which we consider more reliable due to a larger sample of data available, yielded a slightly different value of the diffractive time-scale, $\tau_{\text {diss }}=19.3_{-1.6}^{+1.7} \mathrm{~min}$.

We were also able to analyze the refractive scintillations characteristics for this pulsar. The dynamic spectrum showed characteristic drifting patterns, which are formed by refraction at large scales. We estimated the time-scale of the variation to be $\tau_{\text {riss }}=144 \pm 23 \mathrm{~min}$. The measurement of both scintillation time-scales allowed us to estimate the strength of the scattering parameter as $u=2.7$ and the critical frequency as $5 \mathrm{GHz}$.

We estimated the derived scintillation parameters such as diffractive scale $s_{d}=1.25 \times 10^{8} \mathrm{~m}$, Fresnel scale $r_{F}=$ $6.8 \times 10^{8} \mathrm{~m}$, diffractive angle $\theta_{d}=0.065 \mathrm{mas}$, refractive scale $s_{r}=3.71 \times 10^{9} \mathrm{~m}$ and refractive angle $\theta_{r}=0.005$ mas.

Addition of our results to those available in the literature allowed us to estimate the frequency dependence of the scintillation parameters in the range $74-1700 \mathrm{MHz}$. Its diffractive time-scale varies accordingly to a Kolmogorovtype turbulence spectrum (with either a single thin-screen model or uniform medium distribution), but one may argue that the decorrelation bandwidth does not comply with this. However, since this non-compliance is marginal (see Section 3.1), we believe that PSR B0823+26 may be considered as a source behaving accordingly to Kolmogorov's theory.

Summarizing, we analyzed the largest data sample available so far for this pulsar. This scintillation behaviour of this close-by object may be (marginally) explained by a pure single thin-screen model. However, one has to remember that in our analysis we had no choice but to average our values over an entire observational time span, since some of the parameters (like the refractive time-scale) were measurable only for a few individual sessions. Such approach will definitely average out any interstellar medium inhomogeneity effects, if they are present (such inhomogeneities were observed for other low-DM pulsars; see for example Brisken et al. 2010). This may falsify the results of the scintillation parameters and in turn affect the derived spectral indices. We believe that the observed disappearances of the pulsar are the best proof that indeed the interstellar medium along the PSR B0823+26 line of sight is not homogeneous.

\section{ACKNOWLEDGMENTS}

This paper was supported by the grant DEC2012/05/B/ST9/03924 of the Polish National Science Centre. We are grateful to all staff at the Torun Centre for Astronomy and especially the TCfA observers group, which conducted the observations. Special thanks to the anonymous reviewer for the comments that helped us to improve the quality of this article greatly.

\section{REFERENCES}

Bhat N.D.R., Gupta Y., 2002, ApJ, 567, 342

Bhat N.D.R., Gupta Y., Rao A.P., 1998, ApJS, 500, 262

Bhat N.D.R., Gupta Y., Rao A.P., 1999, ApJS, 514, 249

Bhat N.D.R., Gupta Y., Rao A.P., 1999, ApJS, 121, 483

Brisken W.F., Macquart J.-P., Gao J.J., Rickett B.J., Coles

W.A., Deller A.T., Tingay S.J., West C.J. 2010, ApJ, 708, 232

Clegg A.W., Fey A.L., Lazio T.J.W., 1998, ApJ, 496, 253

Cordes J.M., 1986, ApJ, 311, 183

Cordes J.M., Lazio T.J.W., 2002, ApJ, preprint (arXiv:astro-ph/0207156)

Cordes J.M., Weisberg J.M., Boriakoff V., 1985, A\&pJ, 288, 221

Cordes J.M., Pidwerbetsky A., Lovelace R.V.E., 1986, ApJ, 310, 737

Gupta Y., 1995, ApJ, 451, 717

Gupta Y., Rickett B.J., Coles W.A., 1993, ApJ, 403, 183

Gupta Y., Rickett B.J., Lyne A.G., 1994, MNRAS, 269, 1035

Gwinn C.R., Taylor J.H., Weisberg J.M., Rowlings L.A., 1986, AJ, 91, 338

Hewish A., Wolszczan A., Graham D.A., 1985, MNRAS, 213, 167

Kaspi V.M., Stinebring D.R., 1992, ApJ, 392,530

Konacki M., Lewandowski W., Wolszczan A., Doroshenko, O., Kramer M., 1999, ApJ, 519, L81

Lambert, H.C., Rickett, B.J. 1999, ApJ, 517, 299

Lewandowski W., Kijak J., Gupta Y., Krzeszowski K., 2011, A\&A, 425, 569

Lewandowski W., Dembska, M., Kijak J. and Kowalinska, M, 2013, MNRAS, 434, 69

Lorimer D.R., Kramer M., 2005, Handbook of Pulsar Astronomy, Cambridge Univ. Press, Cambridge

Lyne A.G.,Anderson B., Salter M.J., 1982, MNRAS, 201, 503 
Malofeev V.M., Shishov V.I., Sieber W., Jessner A., Kramer M., Wielebinski R., 1996, A\&A, 308, 180

Rickett B.J., 1990, ARA\&A, 28, 561

Romani, R. W., Narayan R., Blandford R., 1986, MNRAS, 220, 19

Scheuer P.A.G., 1968, Nature, 218, 920

Sieber W., 1982, A\&A, 113, 311

Simonetti J.H., Cordes J.M., Heeschen D.S., 1985, ApJ, 296, 46

Smith F.G., Wright N.C., 1985, MNRAS, 214, 97

Wang N., Manchester R.N., Johnston S., Rickett B., Zhang

J., Yusup A., Chen M., 2005, MNRAS, 358, 270 